\section{Harry Potter and the recessive allele}

SIR - We are bombarded with news of genetic discoveries on an almost daily basis, but people without a formal knowledge of heredity and genetics can have difficulty in deciphering and applying this information. Education and debate across all ages would undoubtedly help, but how can we teach children these concepts?

We believe that successful lessons for younger children can be achieved using analogies of direct interest and relevance. Most children are familiar with J. K. Rowling's stories about the young wizard Harry Potter (whose latest exploit, Harry Potter and the Half-Blood Prince, was published by Bloomsbury in July). They are set in a world like our own, but populated by a minority of people with supernatural powers (wizards and witches) and a majority of people without (muggles).

\section{"With the use of these examples,} the concepts of mendelian genetics can be introduced to children as young as five."

\section{- J. M Craig, R. Dow, M. A. Aitken}

Wizards or witches can be of any race, and may be the offspring of a wizard and a witch, the offspring of two muggles ('muggle-born'), or of mixed ancestry ('half-blood').

This suggests that wizarding ability is inherited in a mendelian fashion, with the wizard allele (W) being recessive to the muggle allele (M). According to this hypothesis, all wizards and witches therefore have two copies of the wizard allele (WW). Harry's friends Ron Weasley and Neville Longbottom and his arch-enemy Draco Malfoy are 'pure-blood' wizards: WW with WW ancestors for generations back. Harry's friend Hermione is a powerful muggle-born witch (WW with WM parents). Their classmate Seamus is a half-blood wizard, the son of a witch and a muggle (WW with one WW and one WM parent). Harry (WW with WW parents) is not considered a pure-blood, as his mother was muggle-born.

There may even be examples of incomplete penetrance (Neville has poor wizarding skills) and possible mutations or questionable paternity: Filch, the caretaker, is a 'squib', someone born into a wizarding family but with no wizarding powers of their own.

We believe that, with the use of these examples, the concepts of mendelian genetics can be introduced to children as young as five, and then built on by gradually introducing specific terms such as 'gene' and 'allele, and relating these to chromosomes and DNA. At every stage, the children's familiarity with the Harry Potter characters can be used as a hook to engage them in discussing concepts of heredity and genetics. Jeffrey M.Craig $\downarrow$, Renee Dow $\uparrow$, MaryAnne Aitkent;

*Chromosome Research, Murdoch Childrens Research Institute, Royal Childrens Hospital, †Genetics Education, Murdoch Childrens Research Institute, Royal Childrens Hospital, † Department of Paediatrics, University of Melbourne, Royal Childrens Hospital,

Flemington Road, Parkville,

Victoria 3052, Australia

\section{Tunnel vision all in the mind at Hothouse High}

SIR - Your News Feature "Hothouse High" (Nature 435, 874-875; 2005) quotes current students of Thomas Jefferson High School for Science and Technology as wishing they had more opportunities to take music and other non-science courses.

As an alumna from the class of 1993 , and a musician, I am dismayed, because this was not the case at all while I attended Jefferson. If anything, there was a strong multidisciplinary approach to teaching, which I believe actually helped those of us who went on to pursue a science career. I disagree with Chris Colin's statement that " tunnel vision at Jefferson hindered thinking about other interests and career choices" and I suggest that the only tunnel vision at Jefferson in danger of affecting students might be their own.

I was saddened by the declaration that two-thirds of the seniors at Jefferson now say they wouldn't choose it again. Perhaps the wrong students are attending Jefferson for the wrong reasons? There is pressure, especially from parents worried about college admissions, for their children to attend Jefferson regardless of their interest in science. Not to mention that Jefferson is a public school, free to attend and free from the violence endemic to most public schools in the greater Washington DC area.

Samantha G.Zeitlin

Department of Medicine, Moores-UCSD Cancer Center, University of Califomia, San Diego,

La Jolla, California 92093, USA

\section{Misconduct: acceptable practices differ by field}

\section{SIR - The USOffice of Science and} Technology Policy wisely states, in its definition of misconduct, that there must be a significant departure from the accepted practices of the relevant research community.

In their Commentary article on the integrity of science, ${ }^{\alpha}$ Scientists behaving badly" (Nature 435, 737-738; 2005), Brian

C. Martinson and colleagues use a survey that blends responses from investigators doing basic discovery science with those doing clinical research, as if the accepted and acceptable practices in these two areas did not differ markedly. However, that is not the case.

For instance, in basic science, there are no specific regulations other than keeping research records (misbehaviour no. 16) for a particular time period. In clinical research, on the other hand, good laboratory practice requires certain types of record-keeping, the omission of which is not just careless but potentially sanctionable, as it would place an investigator out of compliance with Federal regulations.

Also, in basic research, intuition (misbehaviour no. 15) is an important, and perhaps in the end a researcher's best, guide to distinguishing between data and noise. In clinical research, by contrast, intuition should never be used for deciding what data can or cannotbe included in an experiment. Indeed, every subject counts, and excluding subjects without appropriate documentation would be a serious and sanctionable matter.

Finally, Martinson and his colleagues consider "changing the design, methodology or results of a study in response to pressure from a funding source" as a single misbehaviour (misbehaviour no. 10). They ignore the fact that changing research design and methodology - although never the results - is precisely what investigators are supposed to do, to satisfy the criticisms of scientific review groups and the funding agencies that they represent.

Frederick Grinnell

Ethics in Science and Medicine Program, University of Texas Southwestem Medical Center, 5323 Harry Hines Boulevard, Dallas, Texas 75390-9039, USA

\section{Cannabis on a downer}

SIR - Why did you use a down arrow to score the recent US Supreme Court decision banning users from growing cannabis at home ("Scorecard" Nature 435, 724; 2005)?

The issue of medical marijuana in the United States is controversial. Although there is still no clear definitive scientific evidence for either side of the argument, you seemed to pass judgement on this issue in a one-line statement.

Martin J. Neumann

Department of Nuclear, Plasma and Radiological Engineering, College of Medicine,

University of Illinois, Urbana, Illinois 61801, USA

The arrows used in Sidelines are intended to record changes to the current prospects of the topic under discussion, not to pass an editorial judgement, although in this case their meaning was ambiguous News Editor, Nature. 